\title{
Establishment of NOD/SCID mouse model of central nervous system leukemia
}

\author{
DONGFANG LI* ${ }^{*}$ PINGGAN LI* ${ }^{*}$ ZHANWEN HE, ZHE MENG, XIANGYANG LUO and JIANPEI FANG \\ Department of Pediatrics, Sun Yat-Sen Memorial Hospital, Sun Yat-Sen University, Guangzhou, Guangdong 510000, P.R. China
}

Received February 14, 2014; Accepted March 24, 2014

DOI: $10.3892 /$ or.2014.3254

\begin{abstract}
In the present study, we successfully established a NOD/SCID mouse model of central nervous system leukemia by injection of acute monocytic leukemia cell line SHI-1 cells into the lateral ventricle. Immunohistochemistry was used to detect human leukocyte common antigen in brain slices. Nested PCR assay was used to detect MLL/AF6 fusion gene expression. After injection, the condition of the mice gradually progressed to cachexia and death (median survival time, 25 days). Leukemic cells were identified in the lung, bone marrow, and lymph node of one mouse. Brain tissue sections showed invasion into the subdural space, pia mater, arachnoid, along the Virchow-Robin space and into the deep brain parenchyma. In summary, a central nervous system leukemia (CNSL) model was established in NOD/SCID mice.
\end{abstract}

\section{Introduction}

Central nervous system leukemia (CNSL) is a relatively common and severe form of leukemia relapse in clinical practice. The incidence of CNSL in children with acute nonlymphocytic leukemia (ANLL) is $6-29 \%(1,2)$. In the last two decades, considerable progress has been achieved in determining the molecular mechanisms of leukemia, and nonobese-severe immunodeficiency (NOD/SCID) mice have been widely used in hematology research. However, studies on extramedullary infiltration in leukemia are limited, especially studies on this mechanism in CNSL. Furthermore, reports on CNSL are rare, and there is a lack of a convenient and practical ANLL-CNSL model.

Acute monocytic leukemia (FAB classification of M5b) SHI-1 cells have a characteristic $t(6 ; 11)$ (q27;q23) chromosomal translocation and express the MLL/AF6 fusion gene product;

Correspondence to: Dr Jianpei Fang, Department of Pediatrics, Sun Yat-Sen Memorial Hospital, Sun Yat-Sen University, 107 Yanjiang West Road, Guangzhou, Guangdong 510000, P.R. China

E-mail: drfong@yeah.net

${ }^{*}$ Contributed equally

Key words: NOD/SCID, MLL/AF6, leukemia, central nervous system leukemia, SHI-1, Virchow-Robin, acute non-lymphocytic leukemia they have a defect in the p53 gene and their tumorigenicity is high when inoculated subcutaneously in nude mice $(3,4)$. In the present study, we constructed a CNSL model in NOD/SCID mice through intracerebroventricular injection of SHI-1 cells into the lateral ventricle, and in this way established an efficient and reproducible model of CNSL that could be used for further study on the mechanism of extramedullary infiltration of leukemia cells and on possible therapies for CNSL.

\section{Materials and methods}

Five to six-week-old NOD/SCID mice were purchased from the Experimental Animal Center of Zhongshan University and adapted to the environment in a specific pathogen-free (SPF) laminar flow cabinet for 3 days. They had free access to food and water except on the day the intraventricular injection was performed. Food, bedding and other substances the mice came into direct contact with underwent irradiation sterilization or high-pressure treatment before use.

Each mouse in the 2 CNSL groups underwent inoculation of $2 \times 10^{4}$ SHI- 1 cells in the right lateral ventricle under sterile conditions. Mice in the control group had intraventricular inoculation of saline under the same conditions. There were $21 \mathrm{NOD} / \mathrm{SCID}$ mice, divided into 3 groups, an experimental group, a survival group and a control group. In the experimental group $(n=10), 2$ mice were sacrificed at day 3 after lateral ventricle injection in order to examine the spread of the SHI-1 cells and 2 mice were sacrificed for the same purpose each week after that until all mice died. Timely treatment was given to moribund mice. In the survival group $(n=8)$, the times after the lateral ventricle injection of leukemic cells that death occurred were recorded. In the control group $(\mathrm{n}=3), 10 \mu \mathrm{l}$ saline was injected into the lateral ventricle, and the health and survival of the mice were monitored.

Cells from the human monocytic leukemia cell line SHI-1 (a gift from Dr Suning Chen at the Jiangsu Institute of Hematology, Suzhou, China) were cultured in Iscove's modified Dulbecco's medium (IMDM) (Gibco, Carlsbad, CA, USA) and $10 \%$ fetal bovine serum (FBS; Hangzhou Evergreen Biological Co., Ltd., Hangzhou, China) at $37^{\circ} \mathrm{C}$ in an incubator with $5 \% \mathrm{CO}_{2}$ and saturated humidity. Cells in the logarithmic growth phase were collected and inoculated into the right ventricle of the mice.

For lateral ventricle injection of SHI-1 cells, mice were deeply anesthetized with $5 \%$ chloral hydrate $(50 \mu \mathrm{l} / 10 \mathrm{~g})$, and 
fixed on a mouse brain stereotaxic apparatus to expose the anterior fontanelle. Right ventricle positioning was adopted and a microsyringe was used to aspirate $10 \mu \mathrm{l}$ cell suspension and insert it into the right lateral ventricle perpendicularly at a location $2 \mathrm{~mm}$ posterior to the anterior fontanelle and $1.0 \mathrm{~mm}$ lateral to the sagittal suture. The depth of the needle insertion was $2 \mathrm{~mm}$ below the skull surface. The cell suspension was injected at a constant flow rate of $1 \mu \mathrm{l} / \mathrm{min}$, and a total of $10 \mu \mathrm{l}$ of cell suspension $\left(2 \times 10^{4}\right.$ cells $)$ was injected. The duration of the injection was $10 \mathrm{~min}$. The needle was then left at the injection site for $5 \mathrm{~min}$ before withdrawal. The needle was rotated while retreating, and the wound was disinfected and the skin sutured after the needle was withdrawn.

Histopathological examination was performed on animals that died in the survival group and in the experimental group, on the 2 mice that were anesthetized and sacrificed each week after inoculation until all mice in this group had died. The following procedures were performed on animals when death or near-death occurred. Various tissues and organs including heart, liver, spleen, lung, kidney and cervical lymph nodes were collected and cryopreserved in liquid nitrogen. The femur was rinsed with IMDM medium several times until the bone mass was transparent. The flushing fluid was collected and centrifuged, and red blood cells were removed using erythrocyte lysis buffer. After centrifugation, cells were fully dissolved in TRIzol medium and stored in a refrigerator at $-80^{\circ} \mathrm{C}$. Nested PCR was used to detect the MLL/AF6 fusion gene in tissues and bone marrow cells (5).

The cranial and spinal skin and subcutaneous tissue were collected and fixed in 10\% neutral formalin for 24-48 h. After decalcification in decalcification solution containing $10 \%$ hydrochloric acid for $24 \mathrm{~h}$, slices were prepared. Five coronal sections (6) were selected for each head sample. The first section was perpendicular above the bilateral eyes. The second section was perpendicular and $0.6 \mathrm{~cm}$ from the first section. The third section was oblique and cut from the posterior fontanel toward the lower left, which was 90 degrees from the horizontal line. The fourth section was perpendicular and $0.2 \mathrm{~cm}$ outside the third section, and the fifth section was perpendicular $0.2 \mathrm{~cm}$ outside the fourth section. All specimens were routinely embedded in paraffin and cut into slices that were $2 \mu \mathrm{m}$ thick. H\&E staining was performed. Immunohistochemical staining was performed to detect expression of human leukocyte common antigen (LCA) in order to assess leukemic cell infiltration.

According to the degree of leukemia cell infiltration into the brain (7), the CNSL was classified into three grades: Grade I, small amount of leukemic cell infiltration was present in the subdural space, the pia mater and arachnoid. Grade II, an extensive and large amount of leukemic cell infiltration was present in the subdural space, the pia mater, and arachnoid, but there was no brain parenchyma infiltration. Grade III, a large amount of leukemic cell infiltration was present in the subdural space, the pia mater and arachnoid and was accompanied by brain parenchyma infiltration.

Data analysis. Continuous data of WBC counts and survival time are presented as mean \pm SD and median and range, respectively. Categorical data for CNSL grading are presented as counts and percentages. Survival curves were constructed by

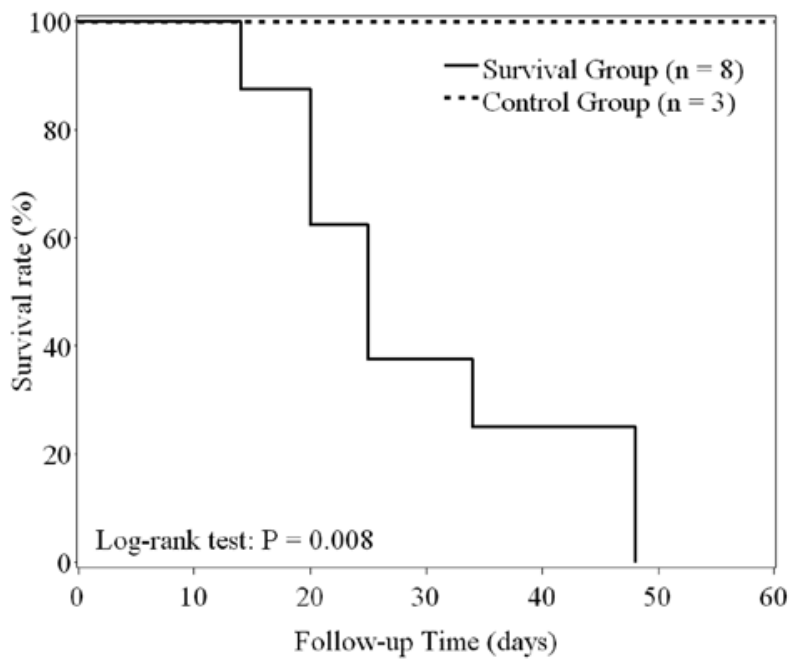

Figure 1. Kaplan-Meier survival curves for NOD/SCID mice in survival and control groups.

the Kaplan-Meier method and compared by the log-rank test. Differences in peripheral WBC counts between the survival and control groups were detected by independent t-test each week from the 1st to the 5 th week. The association between CNSL grading and survival time ( $\leq 20$ vs. $>20)$ was evaluated by Fisher's exact test. Statistical analyses were performed with SAS software version 9.2 (SAS Institute Inc., Cary, NC, USA). A two-tailed $\mathrm{P}<0.05$ indicated a statistically significant result.

\section{Results}

Survival time. Three groups of NOD/SCID mice were included in the present study: an experimental group $(n=10)$, a survival group $(n=8)$ and a control group $(n=3)$. Mice in the experimental group were sacrificed at the following time-points: one mouse on day 3 and 2 mice on days 7, 14 and 21. Three mice in the experimental group died naturally, one mouse on days 24,26 and 31.

In the survival group, the median survival time was 25 days (range, 14-48 days). In the control group, all mice survived more than 60 days. The Kaplan-Meier survival curves (Fig. 1) indicated that survival time was significantly shorter in the survival than in the control group (log-rank test, $\mathrm{P}=0.008)$. The Kaplan-Meier estimate for the 30-day survival rate for mice in the survival group was $37.5 \%$ (95\% confidence interval, 8.7-67.4\%).

Changes in peripheral WBC counts over time. Blood specimens were obtained from tail vein and WBC counts were measured on the first day of each week for five weeks for mice in both the survival and control groups. At each corresponding time-point, the control group showed slightly higher average WBC counts than the survival group (Fig. 2), but this difference did not reach statistical significance (all $\mathrm{P}>0.05$ ). No human leukemia cells were observed in Wright-stained peripheral blood smears. Mice that had not been inoculated with SHI-1 cells were generally in good condition, and no deaths occurred in this group during the 60 days after their saline inoculation. 


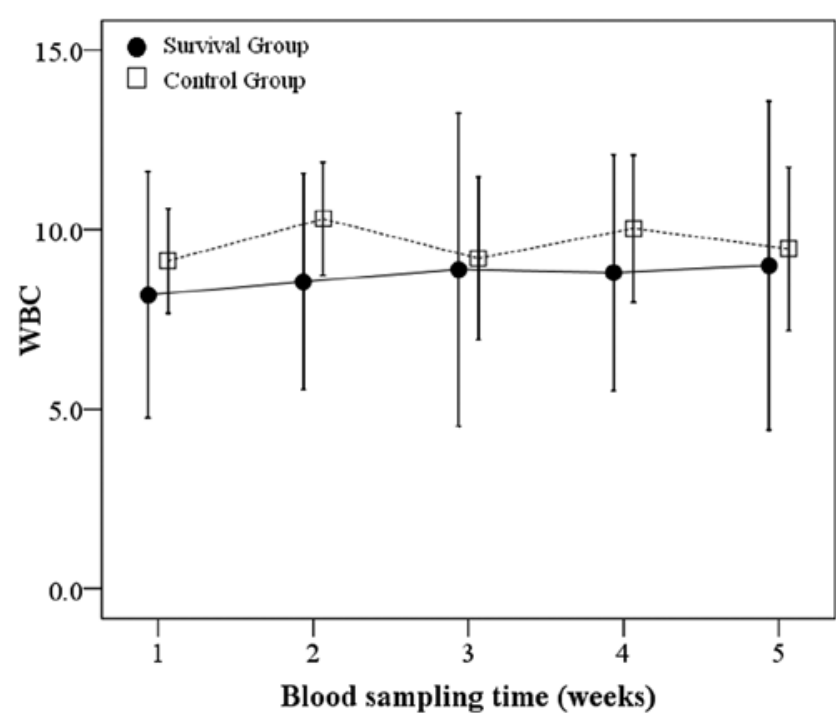

Figure 2. WBC counts $\left(x 10^{6}\right)$ of NOD/SCID mice in survival and control groups at times from 1 to 5 weeks. Data are presented as mean \pm SD. The sample size for the survival group decreased gradually with time due to death, resulting in $8,8,7,5$ and 3 rats in weeks $1,2,3,4$ and 5, respectively. The sample size of the control group was 3 for all time-points $(n=3)$.

CNSL grading. CNSL grading for mice in the experimental and survival groups is shown in Table I. The proportion of mice classified as CNSL grade III in the experimental and survival groups was 50\% (5/10) and 87.5\% (7/8), respectively. No significant association was found between CNSL grading and survival time in either group (all $\mathrm{P}>0.05$ ). With an increase in survival time, CNSL symptoms were aggravated and the degree of brain tissue infiltration worsened. The maximum survival time was 48 days.

Autopsy of the mice did not show any tumor masses in the abdominal cavity, head and face or torso. CNSL cell infiltration was clearly visible and bone mass destruction in the skull and vertebrae and a large amount of leukemia cell infiltration into the vertebral bone marrow cavity were present. The leukemia cells were able to break through the bone mass and infiltrate outside or infiltrate the spinal parenchyma inward. Pathological examination of the head tissue showed a small amount of leukemic cell infiltration in the intracranial arachnoid and the pia mater as early as week 1 . By day 21 , the degree of tumor cell infiltration in brain tissue was severe, and a large amount of leukemia cell infiltration in the subdural space, arachnoid and the pia mater was accompanied by cerebral parenchymal infiltration. Some lumpy masses formed, resulting in compression of the surrounding tissue.

In the medullary cavity of the skull, a considerable amount of leukemia cell infiltration and bone mass destruction were seen, but no lumps had formed on the surface of the skull or in the surrounding soft tissue. Intracranial infiltration occurred mostly in the subdural space, arachnoid and pia mater, and was distributed along the Virchow-Robin space of the blood vessels on the surface of the pia mater. Leukemia cells had attached to the cerebrum, cerebellum and on the surface of the fissures extensively. A large number of leukemia cells were seen to infiltrate from the surface of the cortex to the deep parenchyma in grade III mice. The normal brain tissue
Table I. The distribution of CNSL grades in NOD/SCID mice in experimental and survival groups, according to survival time.

\begin{tabular}{llll}
\hline & \multicolumn{3}{c}{ CNSL grading } \\
\cline { 2 - 4 } & \multicolumn{1}{c}{ I } & \multicolumn{1}{c}{ II } & III \\
& $\mathrm{n}(\%)$ & $\mathrm{n}(\%)$ & $\mathrm{n}(\%)$ \\
\hline Experimental group $(\mathrm{n}=10)$ & $2(20)$ & $3(30)$ & $5(50)$ \\
Survival time $\leq 20$ days & $2(40)$ & $2(40)$ & $1(20)$ \\
Survival time $>20$ days & $0(0)$ & $1(20)$ & $4(80)$ \\
Survival group $(\mathrm{n}=8)$ & $0(0)$ & $1(12.5)$ & $7(87.5)$ \\
Survival time $\leq 20$ days & $0(0)$ & $0(0)$ & $3(100)$ \\
Survival time $>20$ days & $0(0)$ & $1(20)$ & $4(80)$ \\
\hline
\end{tabular}

structure was damaged, and focal perivascular distribution of leukemia cells was seen in deeper brain tissue. Infiltration of brain parenchyma was accompanied by leukemia cell infiltration in the arachnoid, pia mater and surrounding skull and skull destruction. At other places, such as the nasopharynx and retrobulbar area, a considerable amount of leukemia cell infiltration was found to form tumor-like tissues (Fig. 3).

Expressions of $S$ fusion gene MLL/AF6 in various tissues of $N O D / S C I D$ mice. To further study the times of infiltration of various organs, two mice were sacrificed each week after SHI-1 cell inoculation. RT-PCR was used to detect SHI-1 cellspecific fusion gene MLL/AF6 in various organs. No SHI-1 cell fusion gene was amplified in any organ in weeks 1,2 and 4 . The SHI-1 cell fusion gene was amplified in the lung, bone marrow and cervical lymph nodes in one mouse in week 3 , and the product was $280 \mathrm{bp}$.

Fig. 4 shows PCR amplification results of MLL/AF6 of leukemia cells in NOD/SCID mice. The MLL/AF6 fusion gene was amplified in specimens 11, 13 and 14, in lung, bone marrow and lymph node, respectively, of the same mouse.

\section{Discussion}

In the present study, intracerebroventricular direct injection of leukemic cells in mice was used to construct a model of CNSL. As time after inoculation increased, various symptoms of CNSL appeared, tumor cells gradually spread in the brain and spinal cord and in one mouse, cells spread outside the central nervous system into lymph node, lung and bone marrow. The median survival time was 25 days.

Other mouse models of leukemia have used i.p., i.v. and s.c. injections to introduce leukemic cells. Kamel-Reid et al (8), in an early study using i.p. injection of ALL cells into irradiated SCID mice, reported ALL cells in bone marrow at 4 weeks, but did not see ALL cells in brain or any animal deaths until 12 weeks after injection. Gunther et al (9) using i.v. injection of ALL cells into SCID mice, reported that ALL cells were seen in brain and spinal cord by days 21-23, that all mice developed CNSL, but that leukemic cells were not detected deep within the brain parenchyma. In a study examining the characteristics of SHI-1 cells, (3) s.c. injection in mice produced tumor 

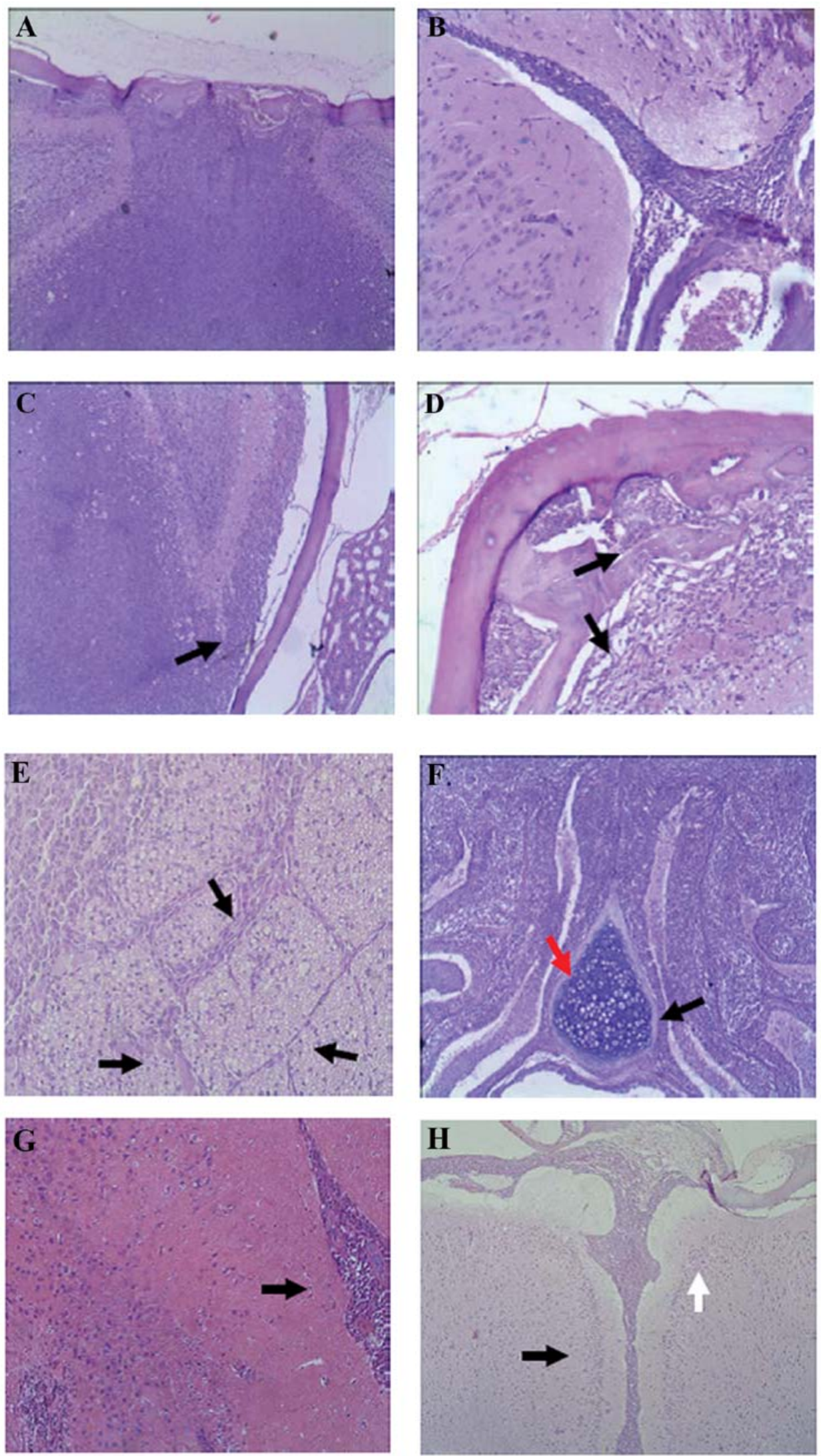

Figure 3. Acute leukemia cell infiltration in the central nervous system. (A and D) A relatively large defect is seen on the inner side of the skull (arrow), and a large number of SHI-1 cells infiltrated the brain parenchyma; they invaded the subdural space, pia mater, and arachnoid from the skull bone marrow, and caused multiple defects on the inner side of the skull (black arrow). (B and H) SHI-1 cells extended deeply along the interhemispheric fissure (black arrow), and local infiltration was seen in the pia mater (white arrow). (C) A considerable amount of SHI-1 cell infiltration was present in the brain parenchyma. Cell infiltration was observed in the pia mater, but no bone mass destruction of the skull was found. (E and G) SHI-1 cells showed invasive growth in the brain, infiltrating the parenchyma along the Virchow-Robin space (black arrow). Multiple perivascular infiltration foci were seen in the deep brain parenchyma. (F) A considerable amount of SHI-1 cell infiltration was seen; the tumor cells entered the sinuses, causing a nasal infiltration defect (black arrow) and forming a local mass (red arrow). 

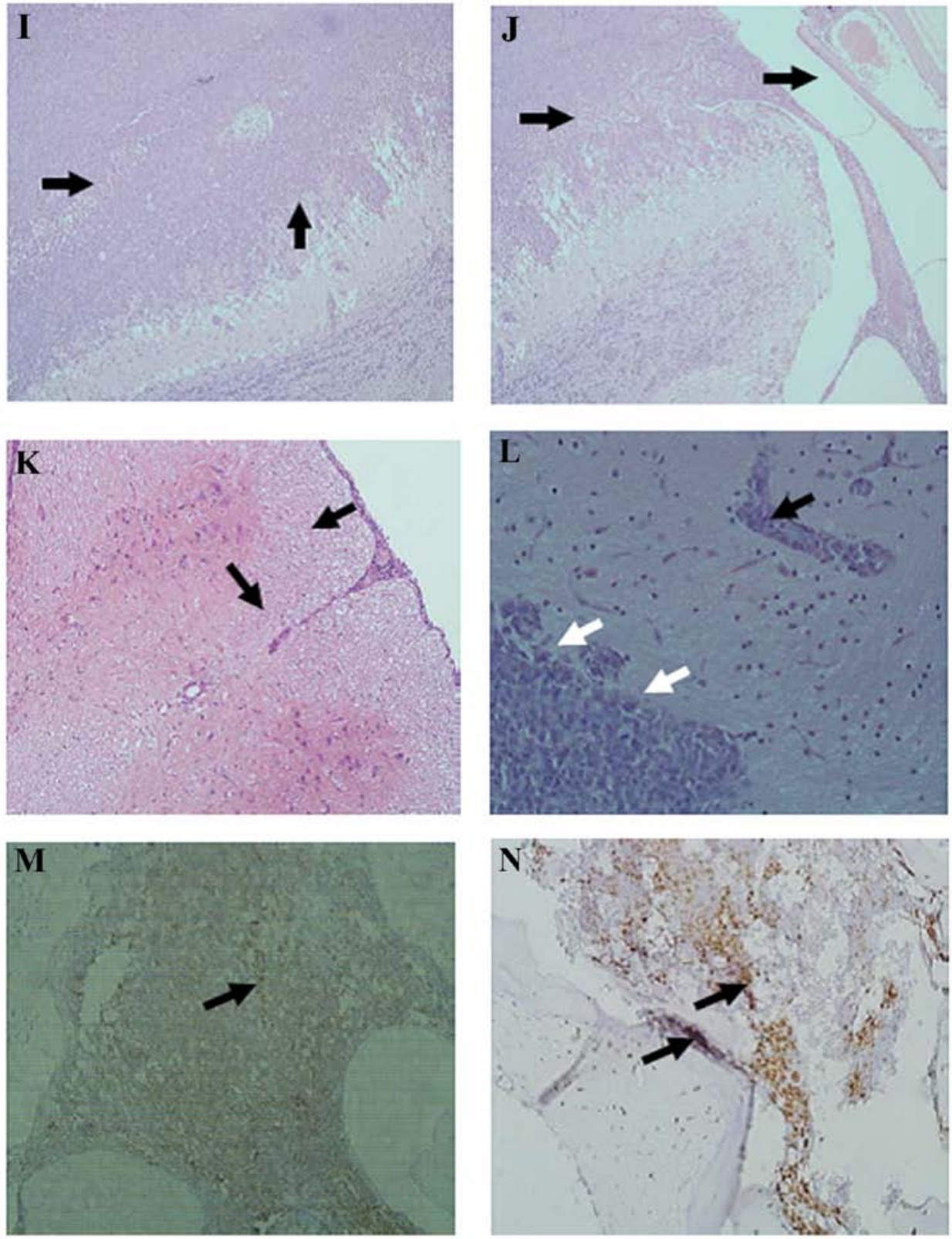

Figure 3. Continued. (I) A large number of SHI-1 cells infiltrated the spinal parenchyma, showing diffuse infiltration (black arrow). (J) SHI-1 cells infiltrated the spinal parenchyma (black arrow) and spinal pia mater, invading the vertebrae and resulting in local bone mass destruction (black arrow). (K) SHI-1 cells extended deeply along the fissures (black arrow) and infiltrated local spinal pia mater (black arrow). (L) SHI-1 cells showed invasive growth in the spinal parenchyma (black arrow) and focal perivascular infiltration (white arrow). (M and N) Immunohistochemical staining showed that the cells infiltrating the brain parenchyma $(\mathrm{M})$ and vertebrae $(\mathrm{N})$ were human LCA-positive cells, indicating that the infiltration was caused by human leukemia SHI-1 cells (black arrow). (A-L) H\&E staining and (M and N) immunohistochemical staining of human LCA. Original magnification, A, C, F, H, I and J, x100; E, L and N, x400; $\mathrm{K}, \mathrm{D}, \mathrm{G}, \mathrm{B}$ and $\mathrm{M}, \mathrm{x} 200$.

masses after 9-19 days. In a study in which SHI-1 cells were injected i.v. into BALB/c nude mice that had been subjected to splenectomy, cytoxan treatment and irradiation, cells were found in the brain in the 3 rd week, and median survival was 41 days.

The human monocytic leukemia cell line SHI-1 originated from mononuclear cells in the bone marrow of an AML-M5b leukemia patient during relapse and is highly invasive (3). Western blot analysis is used to detect EMMPRIN expression in a variety of leukemia cell lines, and all types of leukemia cells have high expressions of EMMPRIN, while SHI-1 has a fixed MLL/AF6 fusion gene. This individual characteristic of the SHI-1 cell line is convenient for experimental tracing. We injected human leukemia cells directly into the lateral ventricle of NOD/SCID mice via a microsyringe to induce direct exogenous CNSL and to establish a CNSL model. Our use of intracerebroventricular injection not only excludes an influence of leukemic cell infiltration in sites outside the bone marrow and central nervous system, but also prevents the harmful effects of the large doses of immunosuppressive agents or preoperative radiation that are required for successful modeling through peripheral injection. Therefore, our model simulates the extramedullary infiltration characteristics of CNSL directly. 


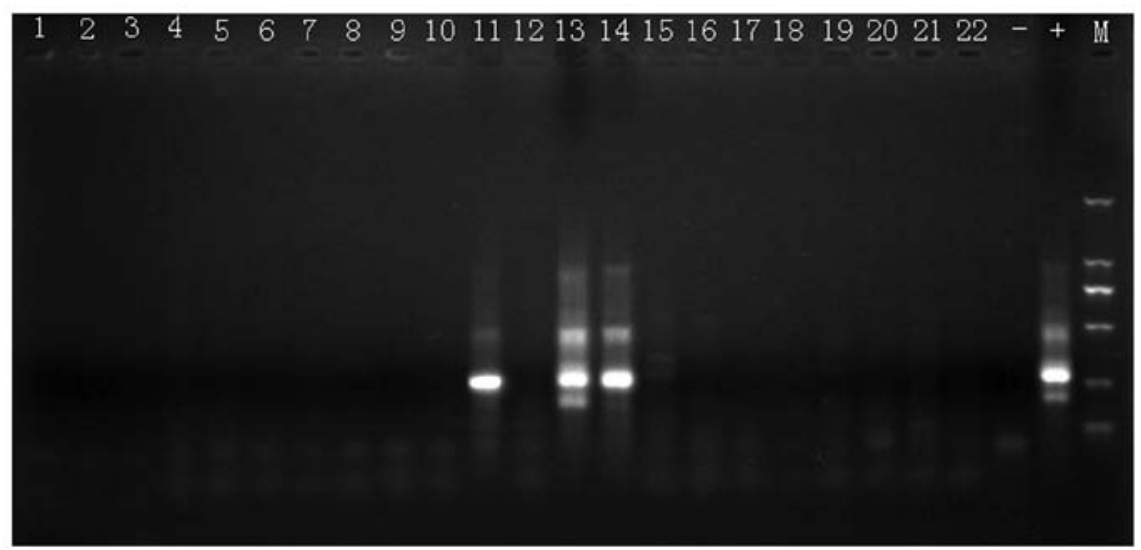

Figure 4. Exemplary PCR amplification of NOD/SCID mouse leukemia cells MLL/AF6. Specimens were loaded in order as lanes 1-7, 8-14 and 15-21 of three mice as heart, liver, kidney, lung, spleen, bone marrow cells and lymph node, respectively. Lane 22 is a heart sample from the fourth mouse. Lanes 23 and 24 are negative control and positive control. The last lane is molecular weight marker with sizes from top to bottom: 2,000, 1,000, 750, 500, 250 and 100 bp. Positive amplified human MLL/AF6 fusion genes were observed in mouse lung (lane 11), bone marrow cells (lane 13) and lymph nodes (lane 14).

In our mouse model, a large amount of cell infiltration was seen in brain tissue, and various degrees of infiltration were also observed in the spinal cord. In addition to nonspecific manifestations including hair thinning, torpor and decreased movement, arched back and reduced dietary intake, obvious lower extremity weakness and paralysis occurred in some mice. However, no convulsions or paralysis of both lower extremities was observed, although convulsions and bilateral lower extremity paralysis have been found in other reports $(7,9)$. Our results indicate that the CNSL model established via lateral ventricle injection of SHI-1 has a more severe and direct impact on brain tissue than models established using peripheral injection. However, it has less or lagging impact on the spinal cord and the spine, which results in more pronounced symptoms of brain damage.

CNSL is a type of childhood leukemia that is difficult to treat and has a high mortality rate. It can occur in the early or late stage during chemotherapy (1). Currently, the chemotherapy regimens advocate early prevention of CNSL; therefore, CNSL often occurs after complete remission in clinical practice and may lead to or be accompanied by bone marrow relapse, eventually leading to chemotherapy failure. The pathogenesis of CNSL has not been fully determined, and it is not fully understood through which pathway tumor cells enter the central nervous system and what factors are involved in tumor cell infiltration into the central nervous system. In terms of the origin of leukemia cells in the central nervous system, there are exogenous and endogenous theories. Most investigators believe in the exogenous theory, namely, that leukemia cells in the central nervous system have come directly from the blood circulation $(10,11)$. However, some investigators believe that in CNSL, leukemia cells are likely to originate in the nervous system itself, since a very small number of hematopoietic stem cells in the choroid or meninges might transform into leukemia cells under the action of certain inducers, and from these locations proliferate and infiltrate and form CNSL (12). Price and Johnson (10) conducted a pathological study on the brain in leukemia patients and considered that leukemia cells enter the brain from the peripheral blood circulation and colonize at the arachnoid, damaging the pia mater and then invading the brain parenchyma. They also thought that the incidence of CNSL might not be related to the blood-brain barrier. Cavallo et al (13) found that leukemic cells infiltrating the skull and the vertebral bone marrow could directly invade the dura, and then invade the arachnoid along the veins between the dura and the arachnoid, finally causing parenchymal infiltration (9).

In the present study, when various tissues and organs of mice were collected for RT-PCR assay, positive results were found in the lymph nodes, lung and bone marrow in one mouse. We applied nested RT-PCR in the present study, and the results had a very high specificity. Our results showed the rationality and possibility of the endogenous doctrine. If leukemic cells spread from the brain tissue to peripheral organs and lymph tissue, the following two situations would occur: i) the cells would infiltrate from the pia mater, pass through the arachnoid and reach the dura, infiltrate local skull tissue and then spread to the whole body; ii) the cells would move from the cerebrospinal fluid and pass through the blood-brain barrier, spread to the blood vessels or enter the lymphatic vessels and lymph nodes, and then spread to the whole body. However, the second situation only occurred in 1 of the 19 mice. Tumor cell infiltration occurred at multiple places in that mouse 3 weeks after lateral ventricle injection, indicating that as time increased, tumor cells proliferated and spread continuously, and that leukemia cells are able to spread from the brain tissue to the periphery. This result is consistent with the sequence of disease progression in clinical practice from CNSL relapse to bone marrow relapse to systemic recurrence. In one mouse, local tissue infiltration occurred and entered the forehead, causing formation of a local tumor mass. This is also consistent with the characteristics of acute myeloid leukemia in that it can easily form local infiltration and chloroma. The results of this study indicate that both of the above-mentioned methods for transfer from inside to outside may occur. Thomas (14) studied human and mouse CNSL and found that the two leukemias have many common characteristics. Leukemia cell stasis, leukemia nodule formation and dura mater and arachnoid hemorrhage in the brain were found in human CNSL. These were also observed in our animal model in the present study. 
The model described in the present study is a stable and repeatable CNSL model that is constructed in NOD/SCID mice using the human acute monocytic leukemia cell line SHI-1. It partially reproduces the progression and infiltration seen in acute monocytic leukemia in human brain tissue, and may provide a relatively ideal experimental animal model for studies on CNSL and on the mechanism of extramedullary infiltration of leukemic cells, treatment of leukemia, molecular mechanisms of leukemia and targeted gene therapy for leukemia.

In conclusion, intracerebroventricular injection of acute monocytic leukemia cell line SHI-1 cells in the lateral ventricle can successfully establish a NOD/SCID mouse model of central nervous system leukemia.

\section{Acknowledgements}

This study was supported by the Medical Scientific and Technology Research Foundation of Guangdong Province, China (B2012106).

\section{References}

1. Johnston DL, Alonzo TA, Gerbing RB, Lange BJ and Woods WG: Risk factors and therapy for isolated central nervous system relapse of pediatric acute myeloid leukemia. J Clin Oncol 23: 9172-9178, 2005.

2. Kobayashi R, Tawa A, Hanada R, Horibe K, Tsuchida M and Tsukimoto I; Japanese Childhood AML Cooperative Study Group: Extramedullary infiltration at diagnosis and prognosis in children with acute myelogenous leukemia. Pediatr Blood Cancer 48: 393-398, 2007.

3. Chen S, Xue Y, Zhang X, Wu Y, Pan J, Wang Y and Ceng J: A new human acute monocytic leukemia cell line SHI-1 with $\mathrm{t}(6 ; 11)$ (q27;q23), p53 gene alterations and high tumorigenicity in nude mice. Haematologica 90: 766-775, 2005.
4. Chen SN, Xue YQ, Zhang XG, Wu YF, Pan JL, Wang Y and Cen JN: Establishment and characterization of a human acute monocytic leukemic cell line, SHI-1, carrying t $(6 ; 11)(\mathrm{q} 27 ; 23)$ and p53 gene alteration. Zhonghua Xue Ye Xue Za Zhi 26: 94-99, 2005 (In Chinese).

5. Mitterbauer G,Zimmer C,Pirc-Danoewinata H, et al: Monitoring of minimal residual disease in patients with MLL-AF6-positive acute myeloid leukaemia by reverse transcriptase polymerase chain reaction. Br J Haematol 109: 622-628, 2000.

6. Zhang H, Fu T, Xing D, et al: Establishment of L615 murine model of meningeal leukemia and preliminary exploration on pathogenesis. Chin J Hematol 4: 335-339, 1983.

7. Li ZJ, Chen ZX, Lu J, Cen JN, He J and Guo LC: Growth and infiltration of human monocytic leukemia cell in nude mice: a model for central nervous system leukemia. Zhonghua Xue Ye Xue Za Zhi: 374-378, 2006 (In Chinese).

8. Kamel-Reid S, Letarte M, Sirard C, et al: A model of human acute lymphoblastic leukemia in immune-deficient SCID mice. Science 246: 1597-1600, 1989.

9. Gunther R, Chelstrom LM, Tuel-Ahlgren L, Simon J, Myers DE and Uckum FM: Biotherapy for xenografted human central nervous system leukemia in mice with severe combined immunodeficiency using B43 (anti-CD19)-pokeweed antiviral protein immunotoxin. Blood 85: 2537-2545, 1995.

10. Price RA and Johnson WW: The central nervous system in childhood leukemia. I. The arachnoid. Cancer 31: 520-533, 1973.

11. Li Z, Chen Z, Lu J, et al: Establishment of a nude mice model of human monocytic leukemia with CNS and multiorgan extramedullary infiltration. Eur J Haematol 77: 128-133, 2006.

12. Sullivan MP: Leukemia infiltration of meninges spinal nerve roots. Pediatrics 32: 63-72, 1963.

13. Cavallo F, Forni M, Riccardi C, et al: Growth and spread of human malignant $\mathrm{T}$ lymphoblasts in immunosuppressed nude mice: a model for meningeal leukemia. Blood 80: 1279-1283, 1992.

14. Thomas LB: Pathology of leukemia in the brain and meninges: postmortem studies of patients with acute leukemia and of mice given inoculations of L1210 leukemia. Cancer Res 25: 1555-1571, 1965. 